\title{
A Quantification Method of Composite Impression of Products by Externalized Evaluation Words of the Appraisal Dictionary with Review Text Data
}

\author{
Sho HASHIMOTO, Atsuhiro YAMADA and Noriko NAGATA
}

Kwansei Gakuin University, 2-1 Gakueni, Sanda-shi, Hyogo 669-1337, Japan

\begin{abstract}
To make users feel a specific impression, a number of studies have provided a way to estimate product impressions with physical features. Although the methodologies of these studies are useful in a wide range of areas, they require a large number of evaluation experiments to reveal the relation between them, and their costs are very high. To solve them, we proposed a method to score each product on the important impression of its domains. This consists of categorizing evaluation terms into internalized and externalized evaluation by using the appraisal dictionary, discovering the important impressions by using only externalized evaluations, and conclusively scoring these product impressions numerically from the term-score and affinity of words. The result of the verification experiment indicated that the obtained scores are valid in terms of their correlations with people's evaluation.
\end{abstract}

Keywords: Quantification, Appraisal dictionary, Review text

\section{INTRODUCTION}

In the field of kansei engineering, construction of a kansei evaluation model that expresses the relationships between impressions and characteristics of objects is one of the main subjects of study [1,2]. Particularly, with the development of technology, in mature industries where it is difficult to differentiate products provided by each company, it has become important to satisfy affective needs in addition to needs such as function and price. Product design aims to make users feel a specific impression that fits and improves the product's image, which satisfies the affective needs [3]. The kansei engineering approach is considered the most reliable and useful methodology to handle users' affective needs [4]. In this approach, there are various levels of impression, from simple impressions that directly represent the physical features of the object such as "soft," to complex impressions such as "sophisticated," which is composed of some simple impressions. For a simple impression, one directly estimates the impression from physical features. On the other hand, for a complex impression, one estimates the impression through the estimation of simpler impressions [5-7]. The kansei engineering approach has been successfully applied in various design domains.

In order to describe the relationship between physical features and impressions, the pairs of scores of the impression of interest and the objects belonging to the product domain of interest must be given as data.
For either regression or other statistical methods, it is necessary to have enough data to use them.

For that purpose, we have traditionally collected data through some experimental procedures targeting humans. They consist of collecting and/or selecting words evaluating impressions, collecting and selecting objects that can be evaluated, and subjective evaluation experiments with selected evaluation words and evaluation objects. In each of these steps, to improve the reliability, these experiments require a number of participants.

Therefore, the researchers have to spend much effort and time creating data sets. For example, in the subjective evaluation experiment using the Semantic Differential (SD) method, time is increased in proportion to the number of stimuli and the number of evaluation words, since the subject is asked to give an observation and an impression with plural evaluation words to a single stimulus. Therefore, it was difficult to obtain impression evaluation data of many stimuli.

In order to solve this problem, we will score the impressions of these products using natural language processing (NLP) for multiple product review corpora.

Many studies have been conducted to estimate the functions and features of products, applying NLP techniques to a review corpus. Htay and Lynn [8] proposed a method to extract opinion words or phrases for each feature by attaining the patterns from the review text through some specific parts of speech (POS) in order to generate summarized information that helps customers make a better selection. Santosh, Babu, Prasad, and 
Vivekananda [9] reported that, by applying the topic model, we can construct a domain-independent Feature Ontology Tree (FOT) in order to identify the characteristics of products. Although most of these methods focused on whether the review is positive or negative, or the main topic of the review, the impressions related to affective evaluation are not yet specifically reated.

In this paper, we work on quantifying the impressions of each product with their reviews, such as in shopping websites, by applying NLP methods. The proposed method is composed of three steps. First, in order to form the collection of evaluation words, we use POS data and Japanese dictionaries of evaluation expressions. Second, using the collection and applying the Hierarchical Dirichlet Process LDA (HDP-LDA) [10], we extract the impression topics. Finally, the scores on the impression topics are calculated by the frequencies of the appearance of evaluation words, term-scores [11] from the result of HDP-LDA, and the similarity between the evaluation words estimated by Word2 Vec [12].

In the next chapter, we give a detailed description of this method.

\section{METHOD}

\subsection{Preprocessing}

As a part of preprocessing, we executed a morphological analysis of the sentences by MeCab [13] (one of a Japanese morphological analyzer) with the dictionary of mecab-ipadic-2.7.0-20070801 [Note 1] and eliminated words other than nouns, verbs, adverbs, adjectives, adnominal words, and prefixes. Then we converted all words into their base form. Moreover, if a word class was a nai adjective base noun, it was concatenated with the following word ("nai"). If a word class was a prefix and the next word class was a noun, we concatenated these words; otherwise, we removed the prefix.

Because of the concern for the accurate deterioration of the model construction due to the orthographic variants, we unified word sets that have the same pronunciation and meaning but different written forms using the morphological analysis. We applied the JUMAN version 7.0 [14] (a Japanese morphological analyzer) for this processing. In JUMAN's dictionary, the tag "daihyo-hyoki" is one of the semantic information of words, and it indicates the representative notation within the set of orthographic variants of the word.

The process of orthographic disambiguation is as follows. Firstly, each word divided by $\mathrm{MeCab}$ is morphologically analyzed using JUMAN. Secondly, when a word is divided into two or more as a result of JUMAN, it is not unified. Otherwise, if a word has "daihyo-hyoki" in the semantic information, the word is unified to the "daihyo-hyoki."

This processing is also executed for dictionaries, and it is introduced in the following section.

\subsection{Collecting and categorizing of evaluation words}

To index the impression of the products, we conducted the following two steps: (1) collecting the evaluation words from all the words, and (2) categorizing the evaluation words into the words related to the individual's feelings/behaviors and the words related to the impression of the product.

In this process, firstly, the words whose POS is a main adjective or noun (adjective base) were collected from all vocabulary of the data. Then, evaluation words of other POS were collected using the following three Japanese dictionaries of evaluation words: EVALDIC ver. 1.0.1 $[15,16]$, Japanese Sentiment Dictionary (Volume of Verbs and Adjectives) ver. 1.0 [16], and Japanese Sentiment Dictionary (Volume of Nouns) ver. 1.0 [17]. Using them, we collected evaluation words that satisfy at least one of the following conditions: (a) It is included in EVALDIC; (b) it has the "hyoka (evaluation)" tag in the Japanese Sentiment Dictionary (Volume of Verbs and Adjectives); and (c) it has the "hyoka (evaluation)" tag in the Japanese Sentiment Dictionary (Volume of Nouns).

Secondly, the collected evaluation words were categorized into the words related to impression of the objects and the words related to the user's feelings/behaviors. Evaluation expressions were roughly classified into two categories: (1) the expressions that represent evaluators' feelings and representative behaviors, such as "enjoy," "get upset," "laugh," or "cry," and (2) the expressions that represent the features of the objects such as "effective," "large," or "sweet." In the previous study [18], the former is called "nai-hyoka" (internalized), and the latter is called "gai-hyoka" (externalized). We categorize the evaluation words based on this definition using the Japanese Appraisal Dictionary attitudinal evaluation ver. 1.0. [19], which categorizes evaluation words as internalized (1730 words; e.g. "delightful (ureshii in Japanese)," "comfortable (kokochiyoi)," or "satisfactory (kiniitta)") or externalized (6815 words; e.g. "guileless (adokenai in Japanese)," "interesting (omoshiroi)", or "cool (ku-ru)"). The evaluation words categorized into both categories such as "satisfied" in the dictionary are categorized as internalized in this paper. The evaluation words categorized into both categories such as "satisfied" are categorized as internalized. 


\subsection{Extracting impression topics}

Firstly, we chose the vocabulary set to make the impression topics. Since internalized expressions of an individual's feelings and behaviors are not impressions of the products, these expressions are inappropriate for the analysis of the relationships between impressions. Therefore, from this step, we use only evaluation words not categorized as internalized. As a result, the words used for learning included only evaluation words of "value" and "impression," that is, evaluation words not included in "affect."

Since it is well known that unsupervised topic models such as Latent Dirichlet allocation (LDA) are useful for product review analysis, we use the Hierarchical Dirichlet Process Latent Dirichlet allocation (HDP-LDA) model [10], which is a nonparametric Bayesian extension of LDA, to extract impression topics. Although LDA is one of the most popular classification methods for texts, the analyst must specify the number of topics, and sometimes the specifying requires professional knowledge of the concerned products. On the other hand, the HDP-LDA has the merit that it is not required to specify the number of topics before the analysis.

To accentuate the difference between each product, all reviews are concatenated for each product. We execute the HDP-LDA for all review sets obtained through the above method. Parameters are estimated by a collapsed Gibbs sampling (CGS) procedure, and the model is run with 500 iterations. Hyper-parameters alpha, beta, and gamma are specified as 2.0, 0.3 and 0.01 , respectively.

\subsection{Calculating impression scores}

The sentences in the review do not contain many complicated grammars, metaphorical expressions, or negations. Also, in the reviews of products with a high score for a certain topic, it is assumed that there are many evaluation words with high importance relative to that topic. Thus, we scored based on the appearance frequency of the evaluation word in the review and the importance of each evaluation word in each topic. In addition, humans potentially feel the similarity of the meaning between impressions. For example, most people feel that "rich" and "luxury" have a somewhat similar meaning. By considering this, it is possible to improve the estimation accuracy of the score of the review in which the target evaluation words are not described. Therefore, we also estimate the similarity between evaluation words automatically and use it for calculating the impression scores.
As the importance of each evaluation word in each topic, term-score was used, as in Yamada, Hashimoto, and Nagata [20]. Term-score is one of the important words in a topic in LDA and in the application of Term Frequency Inverse Document Frequency (TF-IDF), which are used as the importance of words in a document, to the topic. The term-score of word $v$ in topic $k$ is calculated through the following formula:

$$
\text { term-score } k, v=\hat{\beta}_{k, v} \log \left(\frac{\hat{\beta}_{k, v}}{\left(\prod_{j=1}^{K} \hat{\beta}_{k, v}\right)^{\frac{1}{K}}}\right)
$$

where $\hat{\beta}$ is the means of each topic term's probability.

For the calculation of similarity between evaluation words, Word2Vec was used. Word2Vec is a method for automatically vectorizing words based on contextual information around the word. In our scoring method, the input corpus to Word2 Vec includes all reviews of the target domain. After vectorization, we use the cosine similarity between vectors of the word pair as similarity. The detailed settings of Word2Vec are as follows. The dimensionality of the feature vectors is 200 . The window size (maximum distance between the current and predicted word within a sentence) is 5 . The minimum condition of appearance frequency is 20 . The training algorithm is skip-gram with hierarchical softmax.

Overall, the procedure calculates impression scores for each topic of each product in the following way. The score of one review is the mean score of that topic of all reviews. The score of each review is calculated by the appearance frequency of the evaluation words, term-scores, and similarity data. First, for each evaluation word, we multiply the frequency in the review by its term-score in the topic. For words that have a high term-score and are not included in the review, we calculate multiple correlation coefficients and use them as a substitute for the frequency of that word. Multiple correlation coefficient $R^{2}$ is indicated through the following formulas:

$$
\begin{gathered}
R^{2}=\mathbf{c}^{\top} R_{x x}^{-1} \mathbf{c}, \\
\mathbf{c}=\left(r_{x_{1} y}, r_{x_{2} y}, \ldots, r_{x_{N} y}\right)^{\top}, \\
R_{x x}=\left(\begin{array}{cccc}
r_{x_{1} x_{1}} & r_{x_{1} x_{2}} & \cdots & r_{x_{1} x_{N}} \\
r_{x_{2} x_{1}} & \ddots & & \vdots \\
\vdots & & \ddots & \\
r_{x_{N} x_{1}} & \cdots & & r_{x_{N} x_{N}}
\end{array}\right)
\end{gathered}
$$

where $x_{n, y}$, and $r_{x_{n} y}$ are the means of the independent variable, dependent variable, and correlation coefficient between $x_{n}$ and $y$, respectively. In this method, we compute the multiple correlation coefficients between the evaluation word $x_{n}$ that appears in the review and the evaluation 
word $y$ that does not appear. The number of top words that may be calculated in the multiple correlation coefficients must be decided based on the result of impression topics. Finally, we sum scores for each evaluation word, and this value is the score of one review of the topic. Overall, the score of product $p$ of topic $k$ is calculated as the following form:

$$
\begin{array}{r}
\text { Score }_{p k}=\frac{1}{n_{p}} \sum_{r=1}^{n_{p}}\left(\sum_{w \in W_{k}} \text { term-score }_{k w} \cdot f_{r w}\right. \\
\left.+\sum_{t \in T_{r k}} \text { term-score }_{k w} \cdot R_{w}^{2}\right)
\end{array}
$$

where $n_{p}$ is the number of reviews of product $p, W_{k}$ is the set of evaluation words in review $r, f_{r w}$ is the frequency of the word $w$ in review $r$, and $T_{r k}$ is the set of evaluation words that have zero frequency in review $r$ and the top $m$ term-score in topic $k$, respectively. An arbitrary integer $m$ represents how many evaluation words of a topic are emphasized by the analyst.

\section{APPLICATION TO THE WRISTWATCH REVIEW}

\subsection{Data set}

We applied the proposed method to reviews of wristwatches in the Rakuten Data Set [Note 2]. The subject of analysis includes wristwatches that have 10 or more reviews made by purchasers. For each review, we concatenated a review and its title, where the whole number of reviews was 203,086 and the number of products was 4,557 . The total number of words was $4,514,325$, and the total vocabulary size was 19,689 . The number of collected evaluation words was 3,097.

\subsection{Results}

The top words by appearance frequency consisted of the internalized expressions such as "do," "think," "favorite," "buy," and "want" and the externalized expressions such as "good," "satisfaction," "reasonable," "no," and "few." In the proposed method, the latter is applied as the non-affective evaluation word that represents the products' characteristics, and former internalized words are eliminated since these words represent the reviewer's own behaviors or emotions, not products. Consequently, 493 words were categorized as internalized words, and the 2,604 words were categorized as externalized evaluation words.

After eliminating the internalized evaluation words, the number of products comprising 10 or more reviews, including the evaluation words, was 3,644 out of 4,557. As the result of HDP-LDA, 15 topic models were selected, and the perplexity was 243.5. After extracting the impression topics, we set the number of top words to 4 and calculated the impression score. As part of the results, Table 1 shows four topics that represent visual impressions and the top five product images with impression scores of each topic. Since topics consist of words similar in meaning, each topic can be interpreted easily. Also, the result indicates the validity of the discovered topics that represent the impression of the wristwatch. For example, in topic 1 and topic 7, both words "rich" and "elegant" express "high quality", but in addition the latter "elegant" indicates "dignified" or "sophisticated" too. The result shows that heaviness and good texture emphasize impress of "high quality", on the other hand the smallness and delicacy relate impress of "sophisticated". These differences are captured in Table 1, in which Topic 1 relates male high-class watches and Topic 7 relates female high-class watches.

\begin{tabular}{|c|c|c|c|c|c|c|c|}
\hline Topic & Japanese & In English & \multicolumn{5}{|c|}{ Top Images } \\
\hline Topic 1 & $\begin{array}{l}\text { 高級だ, 重い, } \\
\text { 重厚だ, 質感 }\end{array}$ & $\begin{array}{l}\text { rich, heavy, } \\
\text { profound, texture }\end{array}$ & & & & & \\
\hline Topic 6 & $\begin{array}{l}\text { 子供, タフだ, } \\
\text { 丈夫だ, 迅速な }\end{array}$ & $\begin{array}{l}\text { child, tough, } \\
\text { durable, quick }\end{array}$ & & & & & \\
\hline Topic 7 & $\begin{array}{c}\text { 上品だ, 素敵だ, } \\
\text { 小さい, 華奢 }\end{array}$ & $\begin{array}{l}\text { elegant, lovely, } \\
\text { small, delicate }\end{array}$ & 8 & 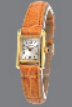 & & 12 & 1 \\
\hline Topic 11 & $\begin{array}{c}\text { 面白い, おしゃれだ, } \\
\text { 変わる,珍しい }\end{array}$ & $\begin{array}{c}\text { funny, fashionable, } \\
\text { strange, novel }\end{array}$ & 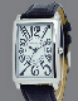 & & & & \\
\hline
\end{tabular}

Table 1: Impression topics and top 5 images of visual impression topics 


\subsection{Subjective evaluation experiment for checking consistency}

To confirm the consistency between the calculated score and people's evaluations, we conducted a subjective evaluation experiment and checked the correlation between the proposed score and mean of evaluation.

\section{- Method}

Twenty participants, 9 women and 11 men, whose ages were between 20 and 24, participated in this experiment. Figure 1 shows an example of the screen display in this experiment. The participants evaluated the 40 wristwatch images using the 7-point Likert-type scale of the SD method. The 40 wristwatches consist of four sets of 10 images that have high scores for one of the topics of the visual impressions. Table 1 shows a part of the set. These images were collected from the website based on product names and product numbers. The image size was unified to $400 \times 400$ pixels, and the height of the wristwatch body was resized to 320 pixels. Moreover, the background colors were unified to the same gray (RGB 128, 128, 128). Four evaluation scales, which indicate conformance to the meaning of each topic, consisted of the top four words of the topic. The 7-point scale ranged from (1) completely disagree (hijoni-atehamaranai) to (7) completely agree (hijoni-atehamaru). Some words were transformed into a suitable form for the experiment. All images were presented in random order by participants.

- Result and discussion

Table 2 shows the correlations between the calculated score and the score given by the human evaluations for each wristwatch. And the table also shows the result of the conventional method [20]. The scores for all visual

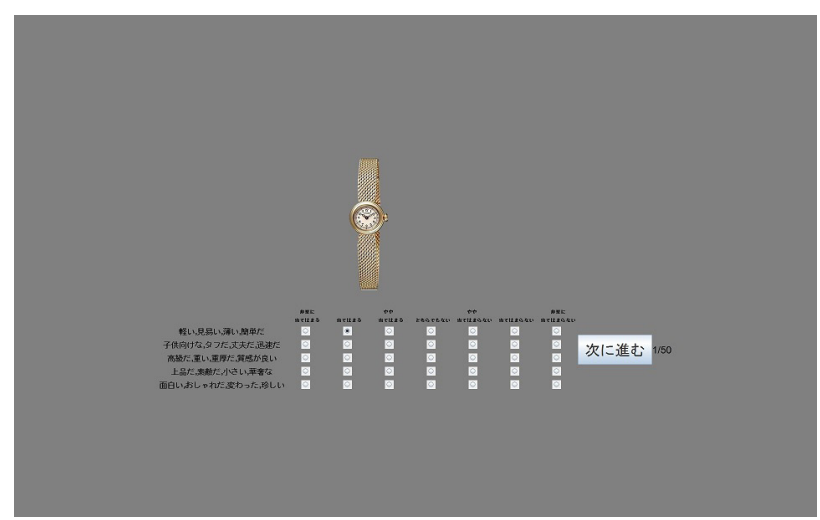

Figure 1: Example of screen display

Table 2: The correlations between the calculated score and human evaluations

\begin{tabular}{|c|c|c|c|c|c|}
\hline Topic & 1 & 6 & 7 & 11 & average \\
\hline \hline Proposed & 0.745 & 0.748 & 0.865 & 0.593 & 0.738 \\
\hline Conventional & 0.690 & 0.766 & 0.841 & 0.612 & 0.727 \\
\hline
\end{tabular}

impression topics, except topic 11, were strongly related to the scores given by the human evaluations, and the score for topic 11 was moderately related. And the mean of four is greater than the result of the conventional method. Therefore, the above result suggests that the proposed method is effective.

We also plotted each wristwatch in Figure 2. The ordinate represents the mean score of human evaluations, and the abscissa represents the calculated score using the proposed method. Each point indicates the wristwatch with the high calculated score. The color of each point indicates which topic has a high calculated score. The calculated scores were normalized to a range between 0 and 1 . These results show that the wristwatches with high calculated scores are highly evaluated by humans in the corresponding topic. Also, as is clearly seen in topic 1 , scores are appropriately even for watches whose impression evaluation is moderate. It seems that the effect considering the similarity between the evaluation words appears.

\section{CONCLUSION}

In this paper, we proposed a method for constructing a kansei evaluation model for product design from review texts without human experiments.

The proposed method consists of three steps. Firstly, evaluation words are collected with three Japanese dictionaries of evaluation expressions and POS data, and they are categorized into the internalized/externalized evaluation tag within Japanese Appraisal Dictionary. Secondly, the impression topics are extracted using HDP-LDA that uses evaluation words other than internalized evaluation words by the above procedure. Lastly, the scores for the topics are calculated with frequencies of evaluation words, its term-scores, and the similarity between the evaluation words estimated by Word2Vec.

We applied the method to the wristwatch reviews of Internet shopping sites. Moreover, we conducted a subjective evaluation experiment on the extracted topics of the impression for comparison with the calculated scores. The results indicated that the scores are well related to subjective evaluation and used to choose the products that can be highly appreciated on the evaluation topics. This indicates that the proposed method is promising as an alternative to the subjective experiment.

We believe that the demand for the automatic construction of a kansei evaluation model will increase because affective needs are increasingly regarded as important in many fields. 


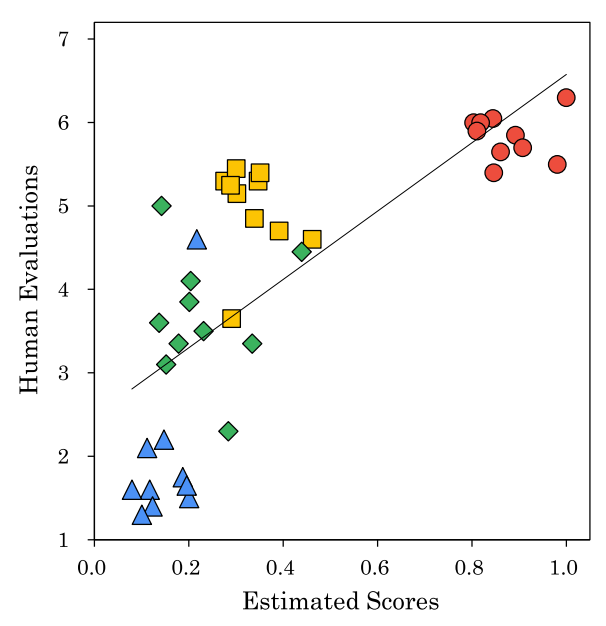

(a) Topic 1

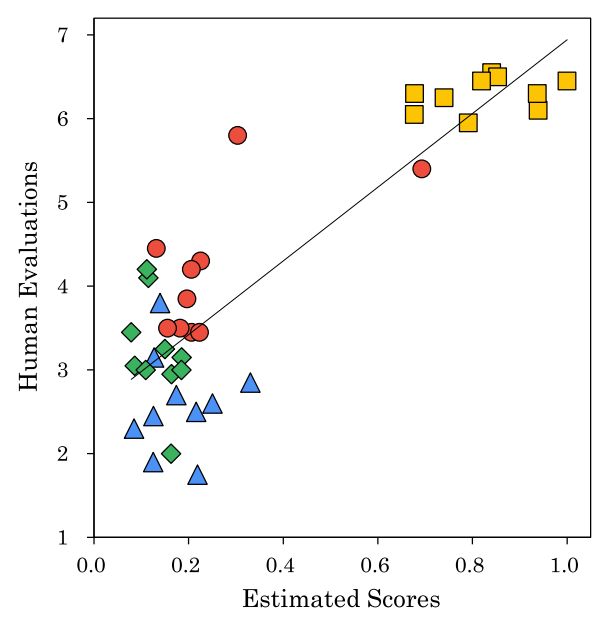

(c) Topic 7

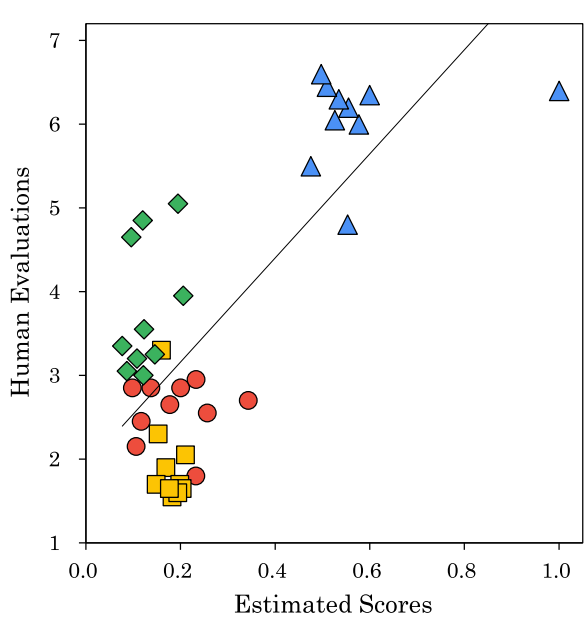

(b) Topic 6

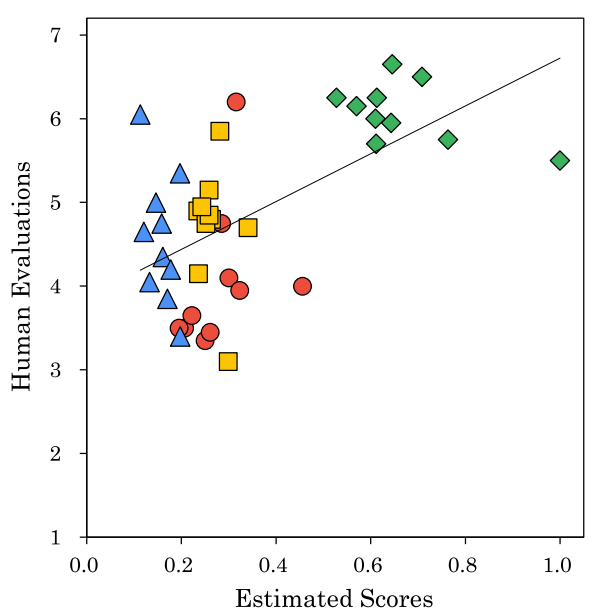

(d) Topic 11
Topic 1

$\triangle$ Topic 6

Topic 7

Topic 11

Figure 2: Scatterplots of each wristwatch

\section{ACKNOWLEDGEMENTS}

This research is partially supported by the Center of Innovation Program from Japan Science and Technology Agency, JST.

\section{NOTES}

1. https://sourceforge.net/projects/mecab/files/mecabipadic/2.7.0-20070801/

2. https://www.nii.ac.jp/dsc/idr/en/rakuten/rakuten.html

\section{REFERENCES}

1. Jing, Y., Ting, J., and Jianbo, X.; A study on interaction design of elderly mobile terminals based on Kansei engineering, 2016 International Conference on Robots \& Intelligent System (ICRIS), pp.59-62, 2016.

2. Aiba, E., Takamatsu, N., Numata, K., Yanagita, S., Suzuki, S., Sato, T., Nagata, N., and Takada, K.; Differences in 'Kansei' space between age groups,
Transactions of Japan Society of Kansei Engineering, 15(7), pp.677-685, 2016. (in Japanese)

3. Wan, Y. J., Li, Y., Li, W. Q., and Yan, X. Q.; Research on method of product innovation based on migrating function, Applied Mechanics and Materials, 278, pp.2269-2273, 2013.

4. Chen, C. H., Khoo, L. P., Chen, K., Pang, J. H., and Huang, Y.; Consumer-oriented product form creation via Kansei engineering, In Proceedings of the International Symposium for Emotion and Sensibility - Emotion Research in Practice, pp.184-191, 2008.

5. Tobitani, K., Matsumoto, T., Tani, Y., Fujii, H., and Nagata, N.; Modeling of the relation between impression and physical characteristics on representation of skin surface quality, The Journal of the Institute of Image Information and Television Engineers, 71(11), pp.J259-J268, 2017. (in Japanese)

6. Nakajima, K., Lee, N., Tobitani, K., Katahira, K., Nagata, N., Shiraiwa, A., Nikata, K., Arakawa, K., 
Ishii, K., Tsukiyama, F., and Kobayashi, S.; Modeling of "High-Class Feeling" on a cosmetic package design, in Proceedings of 22nd Korea-Japan Joint Workshop on Frontiers of Computer Vision (FCV2016), pp.319-324, 2016.

7. Tobitani, K., Matsumoto, T., Tani, Y., and Nagata, N.; Modeling the relation between skin attractiveness and physical characteristics, in Proceedings of the 2018 International Joint Workshop on Multimedia Artworks Analysis and Attractiveness Computing in Multimedia (MMArt\&ACM'18), pp.30-35, 2018.

8. Htay, S. S., and Lynn, K. T.; Extracting product features and opinion words using pattern knowledge in customer reviews, The Scientific World Journal, pp.1-5, 2013.

9. Santosh, D. T., Babu, K. S., Prasad, S. D. V., and Vivekananda, A.; Opinion mining of online product reviews from traditional LDA Topic Clusters using Feature Ontology Tree and Sentiwordnet, International Journal of Education and Management Engineering, 6(6), pp.34-44, 2016.

10. Teh, Y. W., Jordan, M. I., Beal, M. J., and Blei, D. M.; Sharing clusters among related groups: Hierarchical Dirichlet processes, Advances in Neural Information Processing Systems, pp.1385-1392, 2005.

11. Blei, D. M., and Lafferty, J. D.; Topic models, In: Text mining: classification, clustering, and applications, 10(71), p.34, 2009.

12. Mikolov, T., Sutskever, I., Chen, K., Corrado, G. S., and Dean, J.; Distributed representations of words and phrases and their compositionality, Advances in Neural Information Processing Systems, pp.31113119, 2013.

13. Kudo, T.; Mecab: Yet another part-of-speech and morphological analyzer, http://taku910.github.io/mecab (accessed 2019.07.30). (in Japanese)

14. Kurohashi, S.; Improvements of Japanese morphological analyzer JUMAN, in Proceedings of The International Workshop on Sharable Natural Language, pp.22-28, 1994.

15. Kobayashi, N., Inui, K., and Matsumoto, Y.; Designing the task of opinion extraction and structurization, IPSJ SIG Notes, NL171-18, pp.111-118, 2006. (in Japanese)

16. Kobayashi, N., Inui, K., Matsumoto, Y., Tateishi, K., and Fukushima, T.; Collecting evaluative expressions for opinion extraction, Journal of Natural Language Processing, 12(3), pp.203-222, 2005.

17. Higashiyama, M., Inui, K., and Matsumoto, Y.; Learning sentiment of nouns from selectional preferences of verbs and adjectives, in Proceedings of the 14th
Annual Meeting of the Association for Natural Language Processing, pp.584-587, 2008. (in Japanese)

18. Sano, M.; "Nihongo apureizaru hyooka hyoogen jisho -taido hyooka hen-" no koochiku hyooka no tayousei wo toraeru tameno gengoshigen no kaihatsu (The construction of "Japanese dictionary of appraisal -attitude-" Development of language resource to capture diversity of evaluation $\sim$ ), in Proceedings of the 17th Annual Meeting of the Association for Natural Language Processing, pp.115-118, 2010. (in Japanese)

19. Sano, M.; Japanese Dictionary of Appraisal -attitude(JAppraisal Dictionary ver1.0), Gengo Shigen Kyokai, 2010. (in Japanese)

20. Yamada, A., Hashimoto, S., and Nagata, N.; A text mining approach for automatic modeling of Kansei evaluation from review texts, in Proceedings of the 7th International Conference on Kansei Engineering and Emotion Research 2018, pp.319-328, 2018.

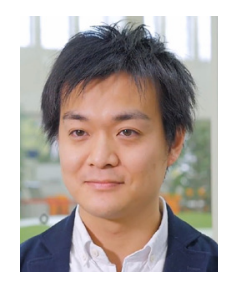

\section{Sho HASHIMOTO (Non-member)}

Sho Hashimoto graduated from the Human Sciences Division, Osaka University in 2007. Completion of doctoral course at Graduate School of Human Sciences, Osaka University in 2015. Doctor (Human Sciences). He joined Kwansei Gakuin University Graduate School of Science and Technology Graduate School of Science and Engineering, as a Doctoral Researcher of the research center for Kansei Value Creation in 2015. Since 2017, he has reached the present position with the research assistant professor at the university science and engineering department. The Behaviormetric Society, Japanese Society of Computational Statistics, Japanese Classification Society etc members.

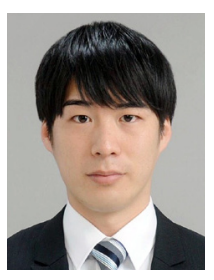

Atsuhiro YAMADA (Non-member)

Atsuhiro Yamada received the BE degree in human system interaction from Kwansei Gakuin Univesity in 2017. He is currently a second year MS student in Science and Technology courses, Kwansei Gakuin University. His research interests include Kansei (affective) information processing and text mining.

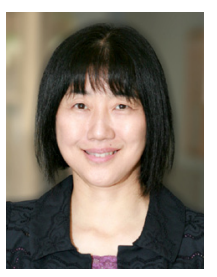

\section{Noriko NAGATA (Member)}

Noriko Nagata received a BS degree in mathematics from Kyoto University in 1983 and a PhD degree in systems engineering from Osaka University in 1996. She was a researcher at the Industrial Electronics and Systems Laboratory of Mitsubishi Electric Corporation from 1983 to 2003. She joined Kwansei Gakuin University in 2003 as an associate professor. She is currently a professor in the department of human system interaction and a director of the research center for Kansei Value Creation. In 2009, she was a visiting scholar at Purdue University. Her research interests include Kansei (affective) information processing, computer graphics, and multimedia systems. She is a member of the IEEE and ACM. 